\title{
High-fidelity manipulation of a qubit enabled by a manufactured nucleus
}

\author{
Justin E. Christensen ${ }^{1 凶}$, David Hucul ${ }^{2}$, Wesley C. Campbell ${ }^{1,3}$ and Eric R. Hudson ${ }^{1,3}$
}

The recently demonstrated trapping and laser cooling of ${ }^{133} \mathrm{Ba}^{+}$has opened the door to the use of this nearly ideal atom for quantum information processing. However, before high-fidelity qubit operations can be performed, a number of unknown state energies are needed. Here, we report measurements of the ${ }^{2} \mathrm{P}_{3 / 2}$ and ${ }^{2} \mathrm{D}_{5 / 2}$ hyperfine splittings, as well as the ${ }^{2} \mathrm{P}_{3 / 2} \leftrightarrow{ }^{2} \mathrm{~S}_{1 / 2}$ and ${ }^{2} \mathrm{P}_{3 / 2} \leftrightarrow{ }^{2} \mathrm{D}_{5 / 2}$ transition frequencies. Using these transitions, we demonstrate high-fidelity ${ }^{133} \mathrm{Ba}^{+}$hyperfine qubit manipulation with electron shelving detection to benchmark qubit state preparation and measurement (SPAM). Using single-shot, threshold discrimination, we measure an average SPAM fidelity of $\mathcal{F}=0.99971(3)$, a factor of $\approx 2$ improvement over the best reported performance of any qubit.

npj Quantum Information (2020)6:35; https://doi.org/10.1038/s41534-020-0265-5

\section{INTRODUCTION}

Quantum error correction allows an imperfect quantum computer to perform reliable calculations beyond the capability of classical computers $^{1-3}$. However, even with the lowest reported error rates ${ }^{4-12}$, the number of qubits $\left(N_{\mathrm{q}}\right)$ required to achieve fault tolerance is projected ${ }^{13}$ to be significantly larger than the state of the art $^{14-16}$. Nonetheless, noisy intermediate-scale quantum (NISQ) devices ${ }^{17}$ are currently being employed to tackle important problems without fault tolerance ${ }^{18-24}$.

For these NISQ devices, single-shot state preparation and measurement (SPAM) infidelity $\left(\epsilon_{\mathrm{s}}\right)$ causes a reduction in computational fidelity that is exponential in qubit number, $\mathcal{F}_{s}=$ $\left(1-\epsilon_{\mathrm{s}}\right)^{N_{\mathrm{q}}}$ (uncorrelated errors). The requirement to perform faithful SPAM therefore limits the number of qubits to $N_{\mathrm{q}}<\ln (2) / \epsilon_{\mathrm{s}}$. While state readout error correction techniques can effectively lower measurement infidelity, they generally require a number of measurements that grows exponentially with $N_{\mathrm{q}}$ and single-shot readout infidelity ${ }^{25}$. For these reasons, and given the desire to increase $N_{\mathrm{q}}$ to tackle problems beyond the reach of classical computers, it is important to develop new means to improve $\epsilon_{\mathrm{s}}$.

The $A=133$ isotope of barium provides a potential path to improving fidelities in atomic ion quantum computing, as this isotope combines the advantages of many different ion qubits into a single system ${ }^{26} \cdot{ }^{133} \mathrm{Ba}^{+}$has nuclear spin $I=1 / 2$, which as we show here, allows fast, robust state preparation and readout of the hyperfine qubit. It possesses both hyperfine and optical $m_{F}=0$ "clock" state qubits, which are relatively insensitive to magnetic fields $\left(m_{F}\right.$ is the projection quantum number of the total angular momentum $F)^{27}$. It also possesses metastable ${ }^{2} D_{\text {, states }}(\tau \sim 1 \mathrm{~min})$, allowing high-fidelity readout, and long-wavelength transitions enabling the use of photonic technologies developed for the visible and near-infrared spectrum. However, before these advantages can be realized, a number of unknown hyperfine and electronic transition frequencies must be determined.

Here, we measure the previously unknown ${ }^{2} \mathrm{P}_{3 / 2}$ and ${ }^{2} \mathrm{D}_{5 / 2}$ hyperfine structure, as well as the ${ }^{2} \mathrm{P}_{3 / 2} \leftrightarrow{ }^{2} \mathrm{~S}_{1 / 2}$ and ${ }^{2} \mathrm{P}_{3 / 2} \leftrightarrow{ }^{2} \mathrm{D}_{5 / 2}$ electronic transition frequencies. Using this knowledge, we demonstrate ${ }^{133} \mathrm{Ba}^{+}$hyperfine qubit manipulation and electron shelving detection. Employing a threshold discrimination and modest fluorescence collection optics (0.28 NA), we measure an average single-shot SPAM fidelity of $\mathcal{F}=0.99971$ (3), the highest reported for any qubit.

In what follows, we first present qubit SPAM using standard hyperfine-selective optical cycling ${ }^{28,29}$ combined with arbitrary qubit rotations and a composite pulse sequence for high-fidelity state transfer. We then present measurement of the unknown hyperfine and electronic transition frequencies. Finally, we use this information to demonstrate high-fidelity SPAM using electron shelving.

\section{RESULTS}

Qubit manipulation and hyperfine-selective SPAM

The hyperfine qubit is defined on the pair of $m_{F}=0$ "clock" states in the ${ }^{2} S_{1 / 2}$ manifold as $|0\rangle \equiv|F=0\rangle$ and $|1\rangle \equiv\left|F=1 ; m_{F}=0\right\rangle$. This hyperfine qubit is initialized to the $|0\rangle$ state after Doppler cooling via optical pumping by applying frequencies $\nu_{493}^{\mathrm{c}}, \nu_{493}^{\mathrm{op}}$, $\nu_{650}^{\mathrm{c}}$, and $\nu_{650}^{\mathrm{sb}}$ (Fig. 1). Rotations of the qubit Bloch vector about $\cos (\phi) \hat{x}+\sin (\phi) \hat{y}$ through angle $\theta, R(\theta, \phi)$, are accomplished by using microwave radiation near $9.925 \mathrm{GHz}^{30}$ controlled by $a$ modular digital synthesis platform ${ }^{31}$. An example rotation of the form $R\left(\Omega_{R} t, 0\right)$ is shown in Fig. 2a, where the average population in state $|1\rangle$ found in 200 trials, measured with a technique described later, is plotted versus the duration of microwave radiation with Rabi frequency $\Omega_{R}=2 \pi \times 57.03(3) \mathrm{kHz}$. The $|1\rangle$ state can be prepared after initialization into $|0\rangle$ by $R(\pi, 0)$; however, we employ a composite pulse sequence, referred to as the CP Robust 180 sequence (attributed to $\mathrm{E}$. Knill) ${ }^{32}$, consisting of the five $\pi$ pulses $R\left(\pi, \frac{\pi}{6}\right) R(\pi, 0) R\left(\pi, \frac{\pi}{2}\right) R(\pi, 0) R\left(\pi, \frac{\pi}{6}\right)$. As shown in Fig. 2b, c, the broad flat features in both curves near zero detuning and $\theta=\pi$ demonstrate resiliency to both pulse area and detuning errors as compared to single $\pi$-pulses, enabling robust day-to-day operation.

Typically, for nuclear spin-1/2 hyperfine qubits, single-shot state readout is accomplished via hyperfine-selective optical cycling $\left(\nu_{493}^{c}\right.$ and $\nu_{650}^{c}$ in Fig. 1) and collection of any resulting fluorescence.

\footnotetext{
'Department of Physics and Astronomy, University of California-Los Angeles, Los Angeles, CA 90095, USA. ${ }^{2}$ United States Air Force Research Laboratory, Rome, NY 13441, USA.
}

${ }^{3}$ UCLA Center for Quantum Science and Engineering, Los Angeles, CA 90095, USA. ${ }^{凶}$ email: j.eugene.christensen@gmail.com 
The $|0\rangle$ and $|1\rangle$ states are determined by threshold discrimination on the number of collected photons, as an atom in the $|1\rangle$ state scatters many photons, while an atom in the $|0\rangle$ state does $\operatorname{not}^{28,29}$. Using this hyperfine-selective optical cycling for SPAM, we measure the fraction of events in which an ion prepared in the $|0\rangle$ state was determined to be $|1\rangle, \epsilon_{|0\rangle}=3.03(4) \times 10^{-2}$, and the fraction of experiments in which an ion prepared in the $|1\rangle$ state was determined to be $|0\rangle, \epsilon_{|1\rangle}=8.65(9) \times 10^{-2}$. The average SPAM fidelity is defined as $\mathcal{F}=1-\epsilon=1-\frac{1}{2}\left(\epsilon_{|0\rangle}+\epsilon_{|1\rangle}\right)=$ $0.9415(5)$. The fidelity of this technique is limited by offresonant excitation to the $\left|{ }^{2} \mathrm{P}_{1 / 2}, F=1\right\rangle$ manifold during readout, which can decay to either $|0\rangle$ or $|1\rangle$, thereby causing

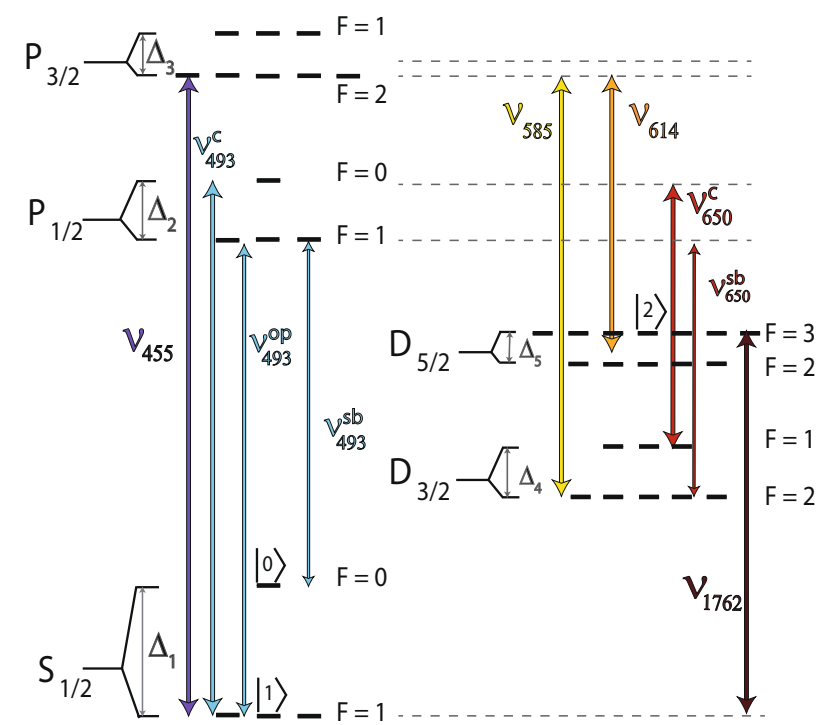

Fig. $1{ }^{133} \mathrm{Ba}^{+}$energy-level diagram and lasers required for laser cooling and high-fidelity SPAM. Laser cooling is accomplished using lasers near 493 and $650 \mathrm{~nm}\left(\nu_{493}^{c}\right.$ and $\left.\nu_{650}^{c}\right)$, and fiber EOMs for repumping sidebands $\left(\nu_{493}^{\mathrm{sb}}\right.$, and $\left.\nu_{650}^{\mathrm{sb}}\right)$. The $|0\rangle$ state is initialized by removing $\nu_{493}^{\text {sb }}$ and adding $\nu_{493}^{\text {op }}$ with the $493 \mathrm{~nm}$ fiber EOM. Microwave radiation at $\Delta_{1} \approx 9.925 \mathrm{GHz}^{30}$ allows for arbitrary rotations on the qubit Bloch sphere. Electron shelving of the $|1\rangle$ state to the metastable ${ }^{2} D_{5 / 2}$ state is accomplished with lasers near 455,585 , and $650 \mathrm{~nm}\left(v_{455}, v_{585}\right.$, and $\left.v_{650}\right)$. A laser near $614 \mathrm{~nm}\left(v_{614}\right)$ is used to depopulate the ${ }^{2} D_{5 / 2}$ manifold after state detection. Measurements from this work include $\Delta_{3}=623(20) \mathrm{MHz}$ and $\Delta_{5}=83(20) \mathrm{MHz}$, with $\Delta_{2}=1840(11) \mathrm{MHz}^{40}$ and $\Delta_{4}=937(20) \mathrm{MHz}^{26}$ previously reported. In future experiments, a laser near $1762 \mathrm{~nm}\left(v_{1762}\right)$ can be used to directly manipulate the optical qubit clock-states $|1\rangle$ and $|2\rangle$. misidentification of the original qubit state ${ }^{28}$. This readout fidelity could be improved with increased light collection efficiency ${ }^{12,33}$.

\section{Spectroscopy}

For high-fidelity SPAM, ${ }^{133} \mathrm{Ba}^{+}$offers another path to state detection. The $|1\rangle$ qubit state can be shelved ${ }^{34}$ to the long-lived ( $\tau \approx 30$ s) metastable ${ }^{2} D_{5 / 2}$ state via the ${ }^{2} D_{5 / 2} \leftrightarrow{ }^{2} S_{1 / 2}$ transition, or optically pumped via the ${ }^{2} \mathrm{P}_{3 / 2}$ state (Fig. 1), followed by Doppler cooling for state readout. Projection into the $|0\rangle$ or $|1\rangle$ state is then determined by threshold discrimination on the number of collected photons, as an atom in the $|0\rangle$ state scatters many photons, while an atom in the ${ }^{2} D_{5 / 2}$ state, indicating $|1\rangle$, does not. Off-resonant scatter is negligible in this case as the Doppler cooling lasers are detuned by many $\mathrm{THz}$ from any ${ }^{2} \mathrm{D}_{5 / 2}$ state transitions.

In principle, shelving of the $|1\rangle$ qubit state is possible via the ${ }^{2} D_{5 / 2} \leftrightarrow{ }^{2} S_{1 / 2}$ electric quadrupole transition near $1762 \mathrm{~nm}\left(v_{1762}\right.$, currently unknown). However, as we demonstrate below, fast, high-fidelity shelving of the $|1\rangle$ state can be achieved with optical pumping by application of the frequencies $v_{455}, v_{585}$, and $\nu_{650}^{c}$ (and $v_{614}$ for deshelving). Of these, only $\nu_{650}^{c}$ has been previously measured $^{26}$. To determine these unknown frequencies, we measure the ${ }^{2} \mathrm{P}_{3 / 2} \leftrightarrow{ }^{2} \mathrm{~S}_{1 / 2}$ and ${ }^{2} \mathrm{P}_{3 / 2} \leftrightarrow{ }^{2} \mathrm{D}_{5 / 2}$ isotope shifts relative to ${ }^{138} \mathrm{Ba}^{+}\left(\delta \nu_{133,138}^{455}\right.$ and $\left.\delta \nu_{133,138}^{614}\right)$ and hyperfine splittings $\Delta_{3}$ and $\Delta_{5}$ (Fig. 1). To measure $\Delta_{3}$ and $\delta \nu_{133,138}^{455}$, the atom is prepared in the $\left|{ }^{2} \mathrm{~S}_{1 / 2} ; F=1\right\rangle$ manifold by optical pumping with $\nu_{650}^{\mathrm{c}}$ and $\nu_{650}^{\mathrm{sb}}$ after Doppler cooling. A tunable laser near $455 \mathrm{~nm}\left(v_{455}\right)$ is applied for $50 \mu \mathrm{s}$. When the frequency is near one of the two allowed transitions, excitation followed by spontaneous emission from the ${ }^{2} \mathrm{P}_{3 / 2}$ with branching ratios ${ }^{35} 0.74,0.23$, and 0.03 to the ${ }^{2} \mathrm{~S}_{1 / 2},{ }^{2} \mathrm{D}_{5 / 2}$, and ${ }^{2} D_{3 / 2}$ states, respectively, optically pumps the ion to the ${ }^{2} D_{5 / 2}$ state. The population remaining in the ${ }^{2} S_{1 / 2}$ and ${ }^{2} D_{3 / 2}$ states is then detected by collecting fluorescence while Doppler cooling and using threshold discrimination on the number of collected photons to decide if the atom was in the ${ }^{2} D_{5 / 2}$ state. This sequence (see Supplementary Information) is repeated 200 times per laser frequency, and the average population is shown Fig. 3a as a function of frequency. From these data, we find $\Delta_{3}=623(20)$ $\mathrm{MHz}$, and $\delta \nu_{133,138}^{455}=+358(28) \mathrm{MHz}$ relative to ${ }^{138} \mathrm{Ba}^{+}$.

To measure $\Delta_{5}$ and $\delta \nu_{133,138}^{614}$, the atom is Doppler cooled and shelved to the ${ }^{2} D_{5 / 2}$ state via one of the ${ }^{2} P_{3 / 2}$ hyperfine manifolds. The $\left|{ }^{2} D_{5 / 2} ; F=2\right\rangle$ manifold is prepared via shelving on the $\left|{ }^{2} \mathrm{P}_{3 / 2} ; F=1\right\rangle \leftrightarrow\left|{ }^{2} \mathrm{~S}_{1 / 2} ; F=1\right\rangle$ transition, as dipole selection rules forbid decay to the $\left|{ }^{2} D_{5 / 2} ; F=3\right\rangle$ state. Similarly, the $\left.{ }^{2} D_{5 / 2} ; F=3\right\rangle$ manifold is prepared by shelving on the $\left|{ }^{2} \mathrm{P}_{3 / 2} ; F=2\right\rangle \leftrightarrow\left|{ }^{2} \mathrm{~S}_{1 / 2}, F=1\right\rangle$ transition, where 0.93 of decays to (a)

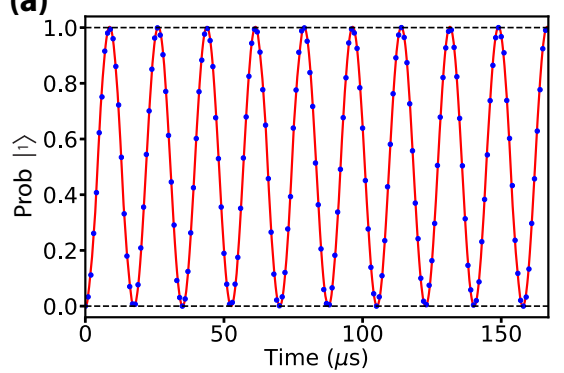

(b)

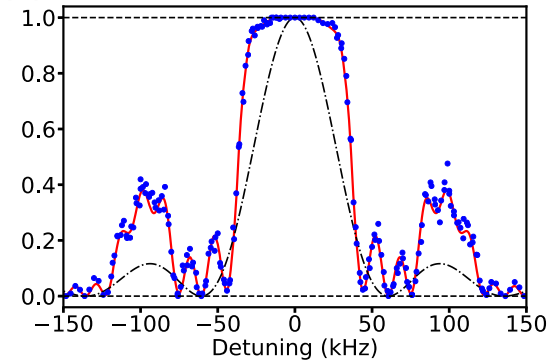

(c)

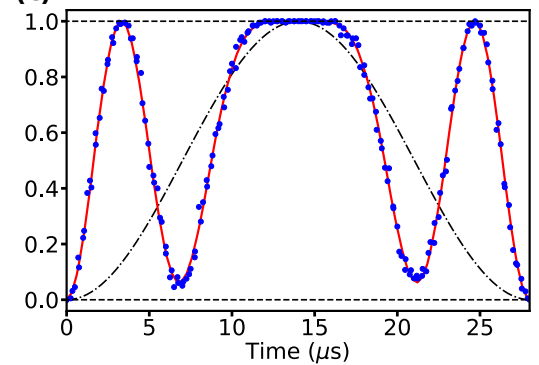

Fig. 2 Microwaves near $9.925 \mathrm{GHz}$ allow for arbitrary rotations on the qubit Bloch sphere. a Probability of shelving $|1\rangle$ after microwave rotations of the form $R\left(\Omega_{R} t, 0\right)$, where $\Omega_{R}=2 \pi \times 57.03(3) \mathrm{kHz}$. b, c To prepare the $|1\rangle$ state, the five $\pi$-pulse CP Robust 180 sequence $R\left(\pi, \frac{\pi}{6}\right) R(\pi, 0) R\left(\pi, \frac{\pi}{2}\right) R(\pi, 0) R\left(\pi, \frac{\pi}{6}\right)$ transfers population from the initially prepared $|0\rangle$ state. b Probability of shelving $|1\rangle$ vs. microwave detuning using the CP Robust 180 sequence with $\Omega_{R}=2 \pi \times 35.4(1) \mathrm{kHz}$. Points are experimental data and solid line represents theoretical prediction for this composite pulse sequence with no fit parameters. c Pulse area $\left(t=\frac{\theta}{\Omega_{R}}\right)$ scan at zero detuning using the CP Robust 180 sequence. Dashed dotted lines in $\mathbf{b}, \mathbf{c}$ are theory for a single $\pi$-pulse, $R(\pi, 0)$. Statistical error bars on individual data points are smaller than markers. 

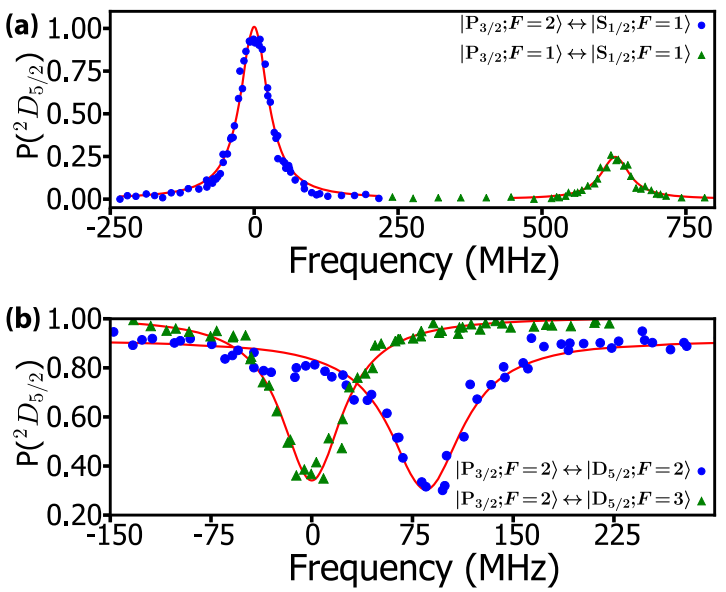

Fig. $3{ }^{2} P_{3 / 2}$ and ${ }^{2} D_{5 / 2}$ hyperfine splitting measurement in ${ }^{133} \mathrm{Ba}+$. a Measurement of the ${ }^{2} \mathrm{P}_{3 / 2}$ hyperfine splitting by tuning the $455 \mathrm{~nm}$ laser. The left (right) peak corresponds to $F=2 \quad(F=1)$ in ${ }^{2} \mathrm{P}_{3 / 2}$. b Measurement of the ${ }^{2} D_{5 / 2}$ hyperfine splitting by tuning the $614 \mathrm{~nm}$ laser. Triangles are data for the $\left.\left.\left.\right|^{2} \mathrm{P}_{3 / 2} ; F=2\right\rangle\left.\leftrightarrow\right|^{2} \mathrm{D}_{5 / 2} ; F=3\right\rangle$ transition, circles are for the $\left|{ }^{2} \mathrm{P}_{3 / 2} ; F=2\right\rangle \leftrightarrow\left|{ }^{2} \mathrm{D}_{5 / 2} ; F=2\right\rangle$ transition. Solid lines are Lorentzian fits. Statistical error bars on individual data points are smaller than markers.

the ${ }^{2} D_{5 / 2}$ are to the $\left|{ }^{2} D_{5 / 2} ; F=3\right\rangle$ manifold. Next, a tunable laser near $614 \mathrm{~nm}$ is applied for $100 \mu \mathrm{s}$. When the frequency is near the $\left|{ }^{2} \mathrm{P}_{3 / 2} ; F=2\right\rangle \leftrightarrow\left|{ }^{2} \mathrm{D}_{5 / 2} ; F=3\right\rangle$ or $\left|{ }^{2} \mathrm{P}_{3 / 2} ; F=2\right\rangle \leftrightarrow\left|{ }^{2} \mathrm{D}_{5 / 2} ; F=2\right\rangle$ transition, spontaneous emission from the ${ }^{2} \mathrm{P}_{3 / 2}$ state quickly deshelves the ion to the $\left|{ }^{2} \mathrm{~S}_{1 / 2} ; F=1\right\rangle$ and ${ }^{2} \mathrm{D}_{3 / 2}$ states. This deshelved population is then detected via Doppler cooling. This sequence is repeated 200 times per laser frequency, and the average population is shown Fig. $3 \mathrm{~b}$ as a function of frequency. From these data, we find the ${ }^{2} D_{5 / 2}$ hyperfine splitting $\Delta_{5}=83(20)$ $\mathrm{MHz}$, and isotope shift $\delta \nu_{133,138}^{614}=+216(28) \mathrm{MHz}$.

High-fidelity SPAM

With the required spectroscopy known, we can calculate the expected fidelity of optically pumped electron shelving detection of the hyperfine qubit as follows. For SPAM of the $|1\rangle$ state, the initial state is prepared as described above, followed by illumination with a laser resonant with the $\left|{ }^{2} \mathrm{P}_{3 / 2} ; F=2\right\rangle \leftrightarrow$ $\left.{ }^{2} \mathrm{~S}_{1 / 2} ; F=1\right\rangle$ transition $\left(v_{455}\right)$ at an intensity below saturation (Fig. 1 and Methods). After excitation of the atom, the ${ }^{2} \mathrm{P}_{3 / 2}$ state quickly $(\tau \approx 10 \mathrm{~ns})$ spontaneously decays to either the ${ }^{2} \mathrm{~S}_{1 / 2},{ }^{2} \mathrm{D}_{5 / 2}$, or ${ }^{2} D_{3 / 2}$ state. Dipole selection rules forbid decay to the $\left.{ }^{2} \mathrm{~S}_{1 / 2} ; F=0\right\rangle(|0\rangle)$ state, resulting in $\mathcal{F}=0.88$ shelving fidelity, limited by population stranded in the ${ }^{2} D_{3 / 2}$ states. To further increase the shelving fidelity, a $650 \mathrm{~nm}$ laser near resonant with the $\left|{ }^{2} \mathrm{P}_{1 / 2} ; F=0\right\rangle \leftrightarrow\left|{ }^{2} \mathrm{D}_{3 / 2} ; F=1\right\rangle$ transition $\left(\nu_{650}^{\mathrm{c}}\right)$, and a laser near $585 \mathrm{~nm}\left(v_{585}\right)$ resonant with the $\left|{ }^{2} \mathrm{P}_{3 / 2} ; F=2\right\rangle \leftrightarrow$ $\left.{ }^{2} \mathrm{D}_{3 / 2} ; F=2\right\rangle$ transition can be applied at an intensity below saturation. The hyperfine structure of ${ }^{133} \mathrm{Ba}^{+}$allows for concurrent repumping of the ${ }^{2} D_{3 / 2}$ states $\left(\nu_{585}\right.$ and $\left.\nu_{650}^{c}\right)$ with all polarization components during the application of $V_{455}$, simplifying the shelving sequence (see Supplementary Information) compared with other species ${ }^{7}$. Dipole selection rules forbid spontaneous emission to the $\left|{ }^{2} \mathrm{~S}_{1 / 2} ; F=0\right\rangle(|0\rangle)$ state resulting in a fidelity of $\mathcal{F} \approx 0.999$. This scheme is limited by off-resonant scatter of $v_{455}$ to the $\left|{ }^{2} \mathrm{P}_{3 / 2} ; F=1\right\rangle$ state, where 0.44 of decays to the ${ }^{2} \mathrm{~S}_{1 / 2}$ are to the $\left.{ }^{2} \mathrm{~S}_{1 / 2} ; F=0\right\rangle$. If $v_{455}$ is linearly polarized parallel to the magnetic field direction ( $\pi$-light), dipole selection rules forbid excitation from the $\left.\left.\left|{ }^{2} \mathrm{P}_{3 / 2} ; F=1 ; m_{F}=0\right\rangle \leftrightarrow\right|^{2} \mathrm{~S}_{1 / 2} ; F=1 ; m_{F}=0\right\rangle$ for the first scattered photon, and the expected fidelity increases to $\mathcal{F}=0.9998$.

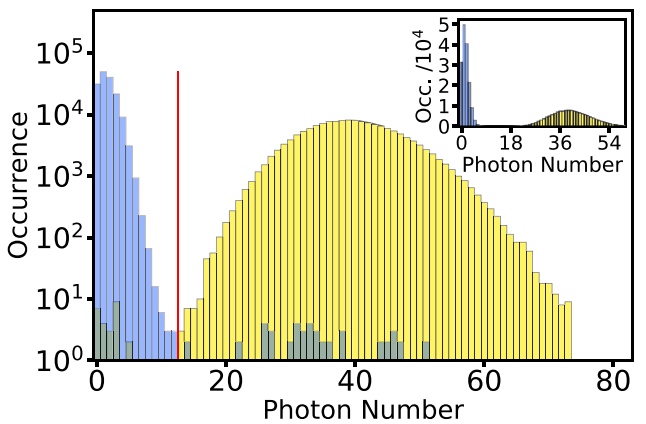

Fig. 4 High fidelity SPAM results for the ${ }^{133} \mathrm{Ba}^{+}$hyperfine qubit. Detection of the $|0\rangle$ (bright) states returns an average of 39 collected photons, while detection of the $|1\rangle$ (dark) state returns an average of 1 collected photon. Using standard spin-1/2 techniques for $|0\rangle$ state preparation $^{28,29}$, a five $\pi$-pulse composite pulse sequence ${ }^{32}$ to prepare the $|1\rangle$ state, and electron shelving for high-fidelity readout, we measure an average SPAM error of $\epsilon=2.9(3) \times 10^{-4}$.

For SPAM of the $|0\rangle$ state, initialization with optical pumping proceeds as described above. After preparation, the $|1\rangle$ state is shelved as previously described, and the state is read out via Doppler cooling. During $|1\rangle$ state shelving, off-resonant excitation to the $\left|{ }^{2} \mathrm{P}_{3 / 2} ; \mathrm{F}=1\right\rangle$ followed by spontaneous emission can shelve the ion to the ${ }^{2} D_{5 / 2}$ state. This results in an expected SPAM fidelity of $\mathcal{F}=0.9998$.

To experimentally test these predictions, state preparation of each qubit state is applied to a single trapped ${ }^{133} \mathrm{Ba}^{+}$ion and read out using the highest fidelity optically pumped shelving scheme (see Methods for experimental parameters). Before each SPAM attempt, the Doppler cooling fluorescence is monitored to determine if an SPAM attempt can be made. If the count rate does not reach a predetermined threshold of $2 \sigma$ below the Doppler cooling mean count rate, chosen before the experiment begins and constant for all SPAM measurements, the subsequent SPAM attempt is not included and deshelving and Doppler cooling are repeated until the threshold is met. Each qubit state is attempted in blocks of 200 consecutive trials, followed by the other qubit state, for a combined total of 313,792 trials. The number of photons detected after each experiment is plotted in Fig. 4, and a threshold at $n_{\text {th }} \leq 12$ photons maximally discriminates between $|0\rangle$ and $|1\rangle$. The fraction of events in which an attempt to prepare the $|0\rangle$ state was measured to be $|1\rangle$ is $\epsilon_{|0\rangle}=1.9(4) \times 10^{-4}$, while the fraction of experiments in which an attempt to prepare the $|1\rangle$ state was measured to be $|0\rangle$ is $\epsilon_{|1\rangle}=3.8(5) \times 10^{-4}$. The average SPAM fidelity is $\mathcal{F}=$ $1-\frac{1}{2}\left(\epsilon_{|0\rangle}+\epsilon_{|1\rangle}\right)=0.99971(3)$.

Table 1 provides an error budget with estimates of the individual sources of error that comprise the observed infidelity. In addition to the previously discussed errors, we have experimentally determined several sources of infidelity. The CP Robust 180 sequence is found to have an error of $\epsilon=9(1) \times 10^{-5}$, determined by measuring the $|1\rangle$ state SPAM infidelity as a function of the number of concatenated CP Robust 180 sequences. The state readout duration is determined by the need to statistically separate the $|0\rangle$ and $|1\rangle$ state photon distributions. Our limited numerical aperture requires detection for $4.5 \mathrm{~ms}$, leading to an error due to spontaneous emission from the ${ }^{2} D_{5 / 2}$ state of $1-\exp \left(\frac{4.5 \times 10^{-3}}{30}\right) \approx 1.5 \times 10^{-4}$. This could be reduced with maximum likelihood methods ${ }^{36,37}$ or higher efficiency light collection ${ }^{33}$. Finally, the readout of the ${ }^{2} S_{1 / 2}$ manifold is limited by background gas collisions, characterized by the preparation and readout fidelities of the ${ }^{2} \mathrm{~S}_{1 / 2}$ and ${ }^{2} \mathrm{D}_{5 / 2}$ manifolds in ${ }^{138} \mathrm{Ba}^{+}$, for which we achieve $\mathcal{F}=0.99997(1)$. 
Table 1. Experimental error budget for state preparation and measurement (SPAM) of the ${ }^{133} \mathrm{Ba}^{+}$hyperfine qubit.

\begin{tabular}{ll}
\hline Process & Average error $\times 10^{-4}$ \\
\hline Initialization to $|0\rangle$ & 0.1 \\
Off-resonant shelving $|0\rangle$ & 1.0 \\
Readout of ${ }^{2} \mathrm{~S}_{1 / 2}$ manifold & 0.1 \\
$|0\rangle \rightarrow|1\rangle \mathrm{CP}$ Robust 180 sequence & 0.5 \\
Shelving $|1\rangle$ & 1.0 \\
Spontaneous decay during readout & 0.7 \\
Total average SPAM error & 3.4 \\
\hline
\end{tabular}

Errors are estimates based on theoretical models and auxiliary experiments. The $|0\rangle$ state SPAM is limited by off-resonant scatter from the laser used for electron shelving. The $|1\rangle$ state electron shelving is limited by the ${ }^{2} \mathrm{P}_{3 / 2}$ hyperfine splitting, where off-resonant scatter can cause spontaneous emission to the $|0\rangle$ state. Spontaneous emission of the ${ }^{2} D_{5 / 2}$ state and preparation of the $|1\rangle$ state via microwaves are the next largest contribution to the $|1\rangle$ state SPAM error.

It should be possible to further improve the fidelity to $\mathcal{F}>0.9999$. Errors due to $|0\rangle \rightarrow|1\rangle$ state transfer and spontaneous emission during readout could be reduced with higher fidelity population transfer and improved light collection efficiency ${ }^{7,33}$. The shelving fidelity could be improved using a pulsed shelving scheme $^{37}$, or by addition of a $1762 \mathrm{~nm}$ transfer step before optical pumping (Fig. 1) in two ways. First, optical-frequency qubit manipulations have been demonstrated (in other species) with a $\pi$-pulse fidelity of $\mathcal{F}=0.99995^{4}$, suggesting that high-fidelity, unitary transfer to ${ }^{2} D_{5 / 2}$ may be possible. Second, even without the narrow-band laser used for coherent transfer on the electric quadrupole transition, a broad-band $1762 \mathrm{~nm}$ laser could be used to saturate the transition to achieve $50 \%$ population transfer. Performing these operations to each of the ten available Zeeman sublevels will transfer the majority of the population to the ${ }^{2} D_{5 / 2}$ state. If either method via $1762 \mathrm{~nm}$ is followed with the optically pumped shelving scheme, we expect a shelving infidelity below $10^{-6}$.

\section{DISCUSSION}

In summary, we report measurements in ${ }^{133} \mathrm{Ba}^{+}$of the ${ }^{2} \mathrm{P}_{3 / 2}$ and ${ }^{2} D_{5 / 2}$ hyperfine splittings and ${ }^{2} \mathrm{P}_{3 / 2} \leftrightarrow{ }^{2} \mathrm{~S}_{1 / 2}$ and ${ }^{2} \mathrm{P}_{3 / 2} \leftrightarrow{ }^{2} \mathrm{D}_{5 / 2}$ transition frequencies, which are required for high-fidelity state readout and optical qubit manipulations. Using these measurements, we have demonstrated operation of the ${ }^{133} \mathrm{Ba}^{+}$hyperfine qubit, including use of the CP Robust 180 composite pulse sequence, to realize an average single-shot SPAM error of $\epsilon_{\mathrm{s}}=2.9$ (3) $\times 10^{-4}$ via threshold discrimination. This represents $a \approx 2 \times$ reduction of SPAM error for any qubit ${ }^{7}$, and is sufficient for singleshot, projective readout of a register of $\approx 2000$ individually resolved qubits.

\section{METHODS}

We trap and laser cool ${ }^{133} \mathrm{Ba}^{+}$ions as described in ref. ${ }^{26} .{ }^{133} \mathrm{Ba}^{+}$ions are loaded into a linear Paul trap $\left(\omega_{\mathrm{sec}} \approx 2 \pi \times 100 \mathrm{kHz}\right)$ by laser ablating an enriched $\mathrm{BaCl}_{2}$ salt (see Supplementary Notes) deposited on a platinum substrate. Laser cooling is accomplished using external cavity diode lasers (ECDLs) near 493 and $650 \mathrm{~nm}$ detuned approximately half an atomic linewidth from the resonant transitions $\left(\nu_{493}^{c}\right.$ and $\left.\nu_{650}^{c}\right)$ with saturation parameter $s \approx 10$. Fiber electro-optic modulators (EOMs) are used to add repumping sidebands resonant with $\nu_{493}^{\mathrm{sb}}, \nu_{493}^{\mathrm{op}}$, and $\nu_{650}^{\mathrm{sb}}$ (Fig. 1). An applied magnetic field $(B \approx 5 G)$ applied along a radial direction of the ion trap, with laser beams linearly polarized $\approx 45^{\circ}$ from the magnetic field direction, are used to destabilize dark states that result from coherent population trapping $(\mathrm{CPT})^{38}$.
State preparation of the $|0\rangle$ state is accomplished by removing $\nu_{493}^{\text {sb }}$ and adding $\nu_{493}^{\mathrm{op}}$ for $100 \mu \mathrm{s}$ after Doppler cooling. The $|1\rangle$ state is prepared via the CP Robust 180 sequence with approximately $3 \mathrm{~W}$ of microwave power directed with a microwave horn for $\approx 15 \mu \mathrm{s}$.

Electron shelving is accomplished by simultaneously applying three lasers near 455,585 , and $650 \mathrm{~nm}$ for $300 \mu \mathrm{s}$. The ECDL laser near $455 \mathrm{~nm}$ tuned resonant with the $\left|{ }^{2} \mathrm{P}_{3 / 2} ; F=2\right\rangle \leftrightarrow\left|{ }^{2} \mathrm{~S}_{1 / 2} ; F=1\right\rangle$ transition $\left(v_{455}\right)$ is linearly polarized parallel to the magnetic field ( $\pi$-light) with saturation parameter $s \approx 1 \times 10^{-3}$. The ECDL near $1171 \mathrm{~nm}$ frequency doubled using a periodically poled lithium niobate (PPLN) waveguide is tuned resonant with $\left.\left.\left|{ }^{2} \mathrm{P}_{3 / 2} ; F=2\right\rangle \leftrightarrow\right|^{2} \mathrm{D}_{3 / 2} ; F=2\right\rangle$ transition $\left(v_{585}\right)$. The laser is linearly polarized $\approx 45^{\circ}$ from the magnetic field direction with saturation parameter $s \approx 1 \times 10^{-2}$. The ECDL near $650 \mathrm{~nm}$ is tuned to the same parameters as Doppler cooling except for the reduction of saturation parameter to $s \approx 1$. Deshelving of the ${ }^{2} D_{5 / 2}$ manifold back to the cooling cycle is accomplished with an ECDL near $1228 \mathrm{~nm}$ frequency doubled with a PPLN waveguide and linearly polarized $\approx 45^{\circ}$ from the magnetic field direction. The frequency is red-detuned approximately $40 \mathrm{MHz}$ from the $\left.\left.\left|{ }^{2} \mathrm{P}_{3 / 2} ; F=2\right\rangle \leftrightarrow\right|^{2} \mathrm{D}_{5 / 2} ; F=2\right\rangle$ transition and applied for $500 \mu \mathrm{s}$ with saturation parameter $s \approx 1$.

State detection is accomplished by collecting only $493 \mathrm{~nm}$ photons for 4.5 ms using a 0.28 NA commercial objective and photomultiplier tube with approximately $15 \%$ quantum efficiency. The 493 and $650 \mathrm{~nm}$ lasers have the same parameters as Doppler cooling during detection. The collection efficiency, laser parameters, ${ }^{2} \mathrm{P}_{1 / 2}$ branching ratio of approximately 3:1, and CPT of the lambda cooling system result in a $493 \mathrm{~nm}$ photon count rate of approximately $10 \mathrm{kHz}$. Background counts of approximately $150 \mathrm{~Hz}$ are dominated by $493 \mathrm{~nm}$ laser scatter from the $493 \mathrm{~nm}$ Doppler cooling beam.

To measure the ${ }^{2} \mathrm{P}_{3 / 2}$ hyperfine splitting and ${ }^{2} \mathrm{P}_{3 / 2} \leftrightarrow{ }^{2} \mathrm{~S}_{1 / 2}$ isotope shift, Doppler cooling followed by optical pumping with $\nu_{650}^{\mathrm{c}}$ and $\nu_{650}^{\mathrm{sb}}$ prepares the $\left|S_{1 / 2} ; F=1\right\rangle$ manifold. A laser near $455 \mathrm{~nm}\left(v_{455}\right)$ is applied for $50 \mu \mathrm{s}$ with saturation parameter $s \approx 1 \times 10^{-3}$. Doppler cooling then determines population in the ${ }^{2} D_{5 / 2}$ states via fluorescence detection, followed by deshelving to reset the ion to the cooling cycle. The sequence is repeated 200 times per frequency, and the frequency scanned over the ${ }^{2} \mathrm{P}_{3 / 2}$ hyperfine splitting. All lasers are linearly polarized $\approx 45^{\circ}$ from the magnetic field direction.

To measure the ${ }^{2} D_{5 / 2}$ hyperfine splitting and ${ }^{2} P_{3 / 2} \leftrightarrow{ }^{2} D_{5 / 2}$ isotope shift, the ${ }^{2} D_{5 / 2} F=3$ or $F=2$ manifold is prepared by Doppler cooling and applying $v_{455}$. A laser near $614 \mathrm{~nm}\left(v_{614}\right)$ is applied for $100 \mu \mathrm{s}$ with saturation parameter $s \approx 1$. Doppler cooling then determines population in the ${ }^{2} D_{5 / 2}$ states via fluorescence detection, followed by deshelving to reset the ion to the cooling cycle. The sequence is repeated 200 times per frequency, and the frequency scanned between the $\left|P_{3 / 2} ; F=2\right\rangle$ and ${ }^{2} \mathrm{D}_{5 / 2}$ hyperfine splitting. All lasers are linearly polarized $\approx 45^{\circ}$ from the magnetic field direction.

All lasers are stabilized via a software lock to a High Finesse WSU-2 wavemeter $^{39}$. Reported hyperfine measurements (see Supplementary Information) include a systematic uncertainty of $20 \mathrm{MHz}$ due to unresolved Zeeman structure. For isotope shifts, the relevant ${ }^{133} \mathrm{Ba}^{+}$centroid frequency is determined from the hyperfine splitting measurements and then compared to measurements of the corresponding ${ }^{138} \mathrm{Ba}^{+}$transition. All ${ }^{133} \mathrm{Ba}^{+}$and ${ }^{138} \mathrm{Ba}^{+}$measurements use the same experimental hardware and wavemeter. Reported isotope shifts include a $28 \mathrm{MHz}$ systematic uncertainty due to wavemeter drift and unresolved Zeeman structure.

\section{DATA AVAILABILITY}

Data are available upon request.

Received: 9 October 2019; Accepted: 2 March 2020; Published online: 14 April 2020

\section{REFERENCES}

1. Steane, A. Quantum computing. Rep. Prog. Phys. 61, 117-173 (1998).

2. Preskill, J. Fault-Tolerant Quantum Computation. Introduction to Quantum computation and Information. 213-269 (1997).

3. Gottesman, D. An introduction to quantum error correction and fault-tolerant quantum computation. Quantum Information Science and Its Contributions to Mathematics, Proceedings of Symposia in Applied Mathematics. 68, 13-58 (2010). 
4. Gaebler, J. P. et al. High-fidelity universal gate set for ${ }^{9} \mathrm{Be}^{+}$ion qubits. Phys. Rev. Lett. 117, 060505 (2016).

5. Hume, D. B., Rosenband, T. \& Wineland, D. J. High-fidelity adaptive qubit detection through repetitive quantum nondemolition measurements. Phys. Rev. Lett. 99, 120502 (2007).

6. Jeffrey, E. et al. Fast accurate state measurement with superconducting qubits. Phys. Rev. Lett. 112, 190504 (2014).

7. Harty, T. P. et al. High-fidelity preparation, gates, memory, and readout of a trapped-ion quantum bit. Phys. Rev. Lett. 113, 220501 (2014)

8. Ballance, C. J., Harty, T. P., Linke, N. M., Sepiol, M. A. \& Lucas, D. M. High-fidelity quantum logic gates using trapped-ion hyperfine qubits. Phys. Rev. Lett. 117 060504 (2016).

9. Bermudez, A. et al. Assessing the progress of trapped-ion processors towards fault-tolerant quantum computation. Phys. Rev. X 7, 041061 (2017).

10. Erhard, A. et al. Characterizing large-scale quantum computers via cycle benchmarking. Nat. Commun. 10, 5347 (2019).

11. Wu, T., Kumar, A., Giraldo, F. \& Weiss, D. S. Stern-gerlach detection of neutralatom qubits in a state-dependent optical lattice. Nat. Phys. 15, 538-542 (2019).

12. Crain, Sea High-speed low-crosstalk detection of a $171 \mathrm{yb}+$ qubit using superconducting nanowire single photon detectors. Commun. Phys. 2, 97 (2019).

13. Fowler, A. G., Mariantoni, M., Martinis, J. M. \& Cleland, A. N. Surface codes: towards practical large-scale quantum computation. Phys. Rev. A 86, 032324 (2012).

14. Wright, K. et al. Benchmarking an 11-qubit quantum computer. Nat. Commun. 10, 5464 (2019).

15. Friis, N. et al. Observation of entangled states of a fully controlled 20-qubit system. Phys. Rev. X 8, 021012 (2018).

16. Debnath, S. et al. Demonstration of a small programmable quantum computer with atomic qubits. Nature 536, 63 (2016).

17. Preskill, J. Quantum computing in the NISQ era and beyond. Quantum 2, 79 (2018)

18. Nam, Y. et al. Ground-state energy estimation of the water molecule on a trapped ion quantum computer. Preprint at https://arxiv.org/abs/1902.10171 (2019).

19. Zhang, J. et al. Observation of a many-body dynamical phase transition with a 53qubit quantum simulator. Nature 543, 217 (2017).

20. Hempel, C. et al. Quantum chemistry calculations on a trapped-ion quantum simulator. Phys. Rev. X 8, 031022 (2018).

21. Gorman, D. J. et al. Engineering vibrationally assisted energy transfer in a trapped-ion quantum simulator. Phys. Rev. X 8, 011038 (2018).

22. Landsman, K. A. et al. Verified quantum information scrambling. Nature 567, 61-65 (2019)

23. Hucul, D. et al. Modular entanglement of atomic qubits using photons and phonons. Nat. Phys. 11, 37 (2015).

24. Kokail, C. et al. Self-verifying variational quantum simulation of lattice models. Nature 569, 355-360 (2019).

25. Shen, C. \& Duan, L.-M. Correcting detection errors in quantum state engineering through data processing. N. J. Phys. 14, 053053 (2012).

26. Hucul, D., Christensen, J. E., Hudson, E. R. \& Campbell, W. C. Spectroscopy of a synthetic trapped ion qubit. Phys. Rev. Lett. 119, 100501 (2017).

27. Wang, Y. et al. Single-qubit quantum memory exceeding ten-minute coherence time. Nat. Photon. 11, 646-650 (2017).

28. Olmschenk, S. et al. Manipulation and detection of a trapped $y^{+}$hyperfine qubit. Phys. Rev. A 76, 052314 (2007).

29. Acton, M. et al. Near-perfect simultaneous measurement of a qubit register. Quantum Inf. Comp. 6, 465 (2006)

30. Knab, H., Schupp, M. \& Werth, G. Precision spectroscopy on trapped radioactive ions: ground-state hyperfine splittings of ${ }^{133} \mathrm{ba}^{+}$and ${ }^{131} \mathrm{ba}^{+}$. Europhys. Lett. 4, 1361 (1987).

31. Pruttivarasin, T. \& Katori, H. Compact field programmable gate array-based pulsesequencer and radio-frequency generator for experiments with trapped atoms. Rev. Sci. Instrum. 86, 115106 (2015).

32. Ryan, C. A., Hodges, J. S. \& Cory, D. G. Robust decoupling techniques to extend quantum coherence in diamond. Phys. Rev. Lett. 105, 200402 (2010).

33. Noek, R. et al. High speed, high fidelity detection of an atomic hyperfine qubit. Opt. Lett. 38, 4735-4738 (2013).
34. Dehmelt, H. G. Bull. Am. Phys. Soc. 20, 60 (1975).

35. Dutta, T., De Munshi, D., Yum, D., Rebhi, R. \& Mukherjee, M. An exacting transition probability measurement-a direct test of atomic many-body theories. Sci. Rep. 6 29772 (2016).

36. Langer, C. High-Fidelity Quantum Information Processing with Trapped lons. Ph.D. Thesis, University of Colorado, Boulder (2006).

37. Myerson, A. H. et al. High-fidelity readout of trapped-ion qubits. Phys. Rev. Lett. 100, 200502 (2008)

38. Berkeland, D. J. \& Boshier, M. G. Destabilization of dark states and optical spectroscopy in zeeman-degenerate atomic systems. Phys. Rev. A 65, 033413 (2002).

39. High Finesse. WS Ultimate 2 User Manual (High Finesse, 2014).

40. Höhle, C., Hühnermann, H., Meier, T., Ihle, H. \& Wagner, R. Nuclear moments and optical isotope shift of radioactive 133ba. Phys. Lett. B 62, 390-392 (1976).

\section{ACKNOWLEDGEMENTS}

This work was supported by the US Army Research Office under award W911NF-18-10097. We thank Anthony Ransford, Christian Schneider, Conrad Roman and Paul Hamilton for helpful discussions. We thank Peter Yu for technical assistance. Any opinions, findings, and conclusions or recommendations expressed in this material are those of the authors and do not necessarily reflect the views of the AFRL.

\section{AUTHOR CONTRIBUTIONS}

J.E.C. and D.H. conducted the experiment. J.E.C., D.H., W.C.C., and E.R.H. analyzed the results. All authors reviewed the manuscript.

\section{COMPETING INTERESTS}

The authors declare no competing interests.

\section{ADDITIONAL INFORMATION}

Supplementary information is available for this paper at https://doi.org/10.1038/ s41534-020-0265-5.

Correspondence and requests for materials should be addressed to J.E.C.

Reprints and permission information is available at http://www.nature.com/ reprints

Publisher's note Springer Nature remains neutral with regard to jurisdictional claims in published maps and institutional affiliations.

Open Access This article is licensed under a Creative Commons Attribution 4.0 International License, which permits use, sharing, adaptation, distribution and reproduction in any medium or format, as long as you give appropriate credit to the original author(s) and the source, provide a link to the Creative Commons license, and indicate if changes were made. The images or other third party material in this article are included in the article's Creative Commons license, unless indicated otherwise in a credit line to the material. If material is not included in the article's Creative Commons license and your intended use is not permitted by statutory regulation or exceeds the permitted use, you will need to obtain permission directly from the copyright holder. To view a copy of this license, visit http://creativecommons. org/licenses/by/4.0/.

(c) The Author(s) 2020 